\title{
Ultrasound-Guided Fenestration of Tendons About the Hip and Pelvis
}

\section{Clinical Outcomes}

\author{
Jon A. Jacobson, MD, Joshua Rubin, MD, Corrie M. Yablon, MD, Sung Moon Kim, MD, \\ Monica Kalume-Brigido, MD, Aishwarya Parameswaran, MS
}

Received January 8, 2015, from the Department of Radiology (J.A.J., J.R., C.M.Y., S.M.K., M.K.-B.) and Michigan Institute for Clinical and Health Research (A.P.), University of Michigan, Ann Arbor, Michigan USA. Revision requested January 30, 2015. Revised manuscript accepted for publication February 11, 2015.

The research reported in this publication was supported by the National Center for Advanced Translational Sciences of the National Institutes of Health, under award number UL1TR000433.

Address correspondence to Jon A. Jacobson, $M D$, Department of Radiology, University of Michigan, 1500 East Medical Center Dr, TC2910L, Ann Arbor, MI 48109-0326 USA.

E-mail:jjacobsn@umich.edu

doi:10.7863/ultra.15.01009
Objectives - Percutaneous ultrasound-guided needle fenestration has been used to treat tendinopathy of the elbow, knee, and ankle with promising results. The purpose of this study was to evaluate the clinical outcome of ultrasound-guided fenestration of tendons about the hip and pelvis.

Methods - After Institutional Review Board approval, a retrospective search of imaging reports from January 1, 2005, to June 30, 2011, was completed to identify patients treated with ultrasound-guided tendon fenestration about the hip or pelvis. Subsequent clinic notes were retrospectively reviewed to determine whether the patient showed marked improvement, some improvement, no change, or worsening symptoms.

Results - The study group consisted of 22 tendons in 21 patients with an average age of 55.8 years (range, 26.7-77.0 years). The treated tendons included 11 gluteus medius ( 9 tendinosis and 2 partial tears), 2 gluteus minimus (both tendinosis), 8 hamstring ( 6 tendinosis and 2 partial tears), and 1 tensor fascia latae (tendinosis). The average interval to clinical follow-up was 70 days (range, 7-813 days). There was marked improvement in $45.5 \%$ (10 of 22 ), some improvement in $36.4 \%$ ( 8 of 22 ), no change in symptoms in $9.1 \%$ ( 2 of 22 ), and worsening symptoms in $9.1 \%$ ( 2 of 22 ). There were no patient variables (age, chronicity of symptoms, sex, tendon, tendinosis versus tear, prior physical therapy, and prior corticosteroid injection) that were significantly different between patients who improved and those who did not. There were no cases of a subsequent tendon tear or infection.

Conclusions - Clinical follow-up after ultrasound-guided fenestration of the gluteus medius, gluteus minimus, proximal hamstring, and tensor fascia latae tendons showed that $82 \%$ of patients had improvement in their symptoms.

Key Words - fenestration; musculoskeletal ultrasound; tendinopathy; tendinosis; tendon; tenotomy

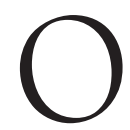
ne cause of chronic pain of the hip and pelvis may be tendinopathy, due to tendon degeneration and a possible tendon tear. ${ }^{1,2}$ Noninvasive treatments for such conditions may include rest, physical therapy with an emphasis on eccentric strengthening, and nonsteroidal anti-inflammatory drugs. ${ }^{3}$ Percutaneous interventional treatments are often considered, which include corticosteroid injection, tendon fenestration, and autologous blood injection, to name a few. ${ }^{4}$

Since the predominant histologic finding with tendinopathy is tendon degeneration and not inflammation, intratendinous corticosteroid injection is typically not indicated; in addition, such injections predispose to tendon rupture. ${ }^{5}$ Corticosteroid injection 
superficial to tendinosis has been shown to reduce pain, although the effects are short lived. ${ }^{5}$ Therefore, tendon fenestration has been considered as a potential treatment option for tendinopathy.

Percutaneous tendon fenestration, also called tenotomy or dry needling, has been used to successfully treat tendinopathy. ${ }^{4}$ Passing a needle through an area of tendinosis theoretically promotes healing through bleeding and inflammation, which results in an increase in local growth factors and other substances. ${ }^{4}$ The most common tendons that have been treated with ultrasound-guided fenestration described in the literature include the common extensor tendon at the elbow, the patellar tendon, and the Achilles tendon. ${ }^{6,7}$ There have been only limited reports that described the use of tendon fenestration beyond these tendons; the effectiveness of fenestration in tendons such as those about the hip and pelvis is unknown. ${ }^{6}$

In our clinical experience, we have been asked to fenestrate various tendons about the body using ultrasound guidance. The purpose of this study was to retrospectively determine the effectiveness of ultrasound-guided tendon fenestration about the hip and pelvis.

\section{Materials and Methods}

After Institutional Review Board approval was obtained, with informed consent waived, the computerized radiology information system was searched from January 1, 2005 , to June 30, 2011, using key words "fenestration," "tenotomy," and "dry needling." Consecutive patients were included if they had a percutaneous ultrasound-guided tendon fenestration of a tendon about the hip or pelvis. Patients' medical records were reviewed, which included subsequent clinic visits to determine whether the symptoms referable to the treated tendon showed marked improvement, some improvement, no change, or worsening symptoms compared to symptoms before the procedure. For a symptom to be categorized as "marked improvement," the dictated clinical report by the referring physician had to have included terms such as "no pain," "complete resolution," and "significant improvement." All other symptom descriptions of improvement were categorized as "some improvement." Patients without any clinical follow-up were excluded.

Ultrasound-guided tendon fenestration was requested by the referring physician as part of routine patient care performed by 1 of 10 fellowship-trained musculoskeletal radiologists with ultrasound and ultrasound-guided procedural experience (range 5-15 years). One of several commercially available ultrasound machines was used (HDI 5000 and iU22, Philips Healthcare, Bothell, WA; and LOGIQ 9 and E9, GE Healthcare, Milwaukee, WI), including both linear and curvilinear transducers. Although the exact details of each patient's procedure were inherently variable given the number of radiologists and the retrospective nature of this study, the following section summarizes the general ultrasound-guided tendon fenestration procedure performed as part of the study.

\section{Fenestration Procedure}

Before the procedure, all patients had avoided nonsteroidal anti-inflammatory medication, including aspirin, for at least 2 weeks so as not to interfere with the inflammatory phase of early tissue healing. The abnormal tendon was confirmed with ultrasound imaging as a target for fenestration. Tendinosis was characterized as abnormal tendon hypoechogenicity; well-defined anechoic clefts or defects were characterized as partial tendon tears. The presence of a full-thickness tear was a contraindication for tendon fenestration.

With a sterile technique and a $1 \%$ lidocaine local anesthetic, a spinal needle ( 20 or 22 gauge) was advanced into the abnormal tendon segment in plane with the ultrasound transducer and sound beam. The needle orientation was either long axis or short axis to the tendon at the operator's discretion but was typically long axis. Lidocaine injection at the surface of the tendon was also at the discretion of the radiologist if needed to reduce symptoms, although direct intratendinous injection was avoided unless deemed necessary. With the inner trocar (or stylet) removed, the needle was repeatedly passed through the abnormal tendon segment. The number of passes through the tendon varied depending on the size of the tendon abnormality, although 20 to 40 passes were typical. Softening of the abnormal tendon after treatment was considered an additional indicator of adequate fenestration. The transducer was turned $90^{\circ}$ and the needle repositioned as necessary to ensure that the entire tendon abnormality was treated. Additional goals included targeting the adjacent bone as well as attempting to break up any intratendinous degenerative calcification, which appears linear unlike the amorphous appearance of hydroxyapatite crystal deposition. After the procedure, the patient was counseled to continue activities of daily living but to avoid strenuous activity related to the pelvis and hip for 2 weeks and any activity that caused pain. The patient was advised to refrain from nonsteroidal anti-inflammatory medication and ice for 2 weeks after the procedure. 


\section{Data Analysis}

Patients' results were evaluated to determine whether there were any significant differences among the various clinical outcomes. Patients were categorized into 2 groups (marked improvement versus other outcome) and compared with respect to demographics (age and sex), site and type of tendon abnormality (tendinosis or tear), chronicity of symptoms, and prior physical therapy or steroid injection, using a Wilcoxon rank sum test (because the continuous variables are not normally distributed) for continuous variables and Fisher exact test for categorical variables. Similarly, patients were categorized into 2 groups (any improvement versus other outcome) and compared. A 5\% level of significance was used to evaluate statistical significance in all analyses. The above analyses were performed with SAS version 9.4 software (SAS Institute Inc, Cary, NC).

\section{Results}

The initial retrospective search yielded 27 patients; however, 6 patients were lost to follow-up. The final study group consisted of 22 tendon fenestrations in 21 patients ( 1 patient had each hip treated) with an average age of 55.8 years (range, 26.6-77.0 years; 16 female and 5 male). Patient symptoms were present an average of 29 months (range, 3.2-105.8 months) before fenestration. Physical therapy was prescribed in $45.5 \%$ (10 of 22), and prior blind corticosteroid injection ( $>6$ months before fenestration) was completed in $36.4 \%$ ( 8 of 22: 7 gluteal and 1 hamstring).

The tendons that were treated with fenestration included the gluteus medius in 50\% ( 11 of $22: 9$ tendinosis and 2 partial tears) (Figure 1), proximal hamstring in $36.4 \%$ ( 8 of 22: 6 tendinosis and 2 partial tears) (Figure 2), gluteus minimus in $9.1 \%$ ( 2 of 22 : both tendinosis) (Figure 3 ), and tensor fascia latae in $4.6 \%$ ( 1 of 22 : tendinosis) (Figure 4). Two patients had total hip arthroplasty (both gluteus medius tendinosis). Two patients had calcification within tendinosis (both proximal hamstring). No patients had bursal distention about the greater trochanter when evaluated with ultrasound. All patients tolerated the procedure without any immediate complications.

The average interval to retrospective clinical followup was 70 days (SD, 168.5 days; range, $7-813$ days). Overall, there was marked improvement in $45.5 \%$ (10 of 22: 5 gluteus medius, 2 gluteus minimus, 2 hamstring, and 1 tensor fascia latae). Some improvement was seen in $36.4 \%$ ( 8 of 22: 5 hamstring and 3 gluteus medius). No change in symptoms was seen in 9.1\% ( 2 of $22: 2$ gluteus medius), and worsening symptoms were seen in 9.1\% ( 2 of $22: 1$ gluteus medius and 1 hamstring).
When categorizing the patients into 2 groups (marked improvement versus other outcome), there was no significant difference with regard to age $(P=.63)$, sex $(P=.35)$, tendon abnormality $(P=.59)$, specific tendon $(P=.17)$, chronicity of symptoms $(P=.32)$, prior physical therapy $(P>.99)$, or prior corticosteroid injection $(P=.67)$. Similarly, when the patients were categorized into 2 groups (any improvement versus other outcome), there was no significant difference with regard to age $(P=.43)$, sex $(P>.99)$, tendon abnormality $(P=.55)$, specific tendon $(P=.79)$, chronicity of symptoms $(P=.43)$, prior physical therapy $(P>.99)$, or prior corticosteroid injection $(P=.60)$.

Figure 1. Images from a 71-year-old woman with gluteus medius tendinosis and fenestration. A, Sonogram in a long-axis orientation to the gluteus medius showing abnormal hypoechoic enlargement of the gluteus medius tendon (arrows) at the superoposterior facet of the greater trochanter (GT). B, Twenty-gauge needle (arrowheads) during fenestration (left side of image is cephalad).
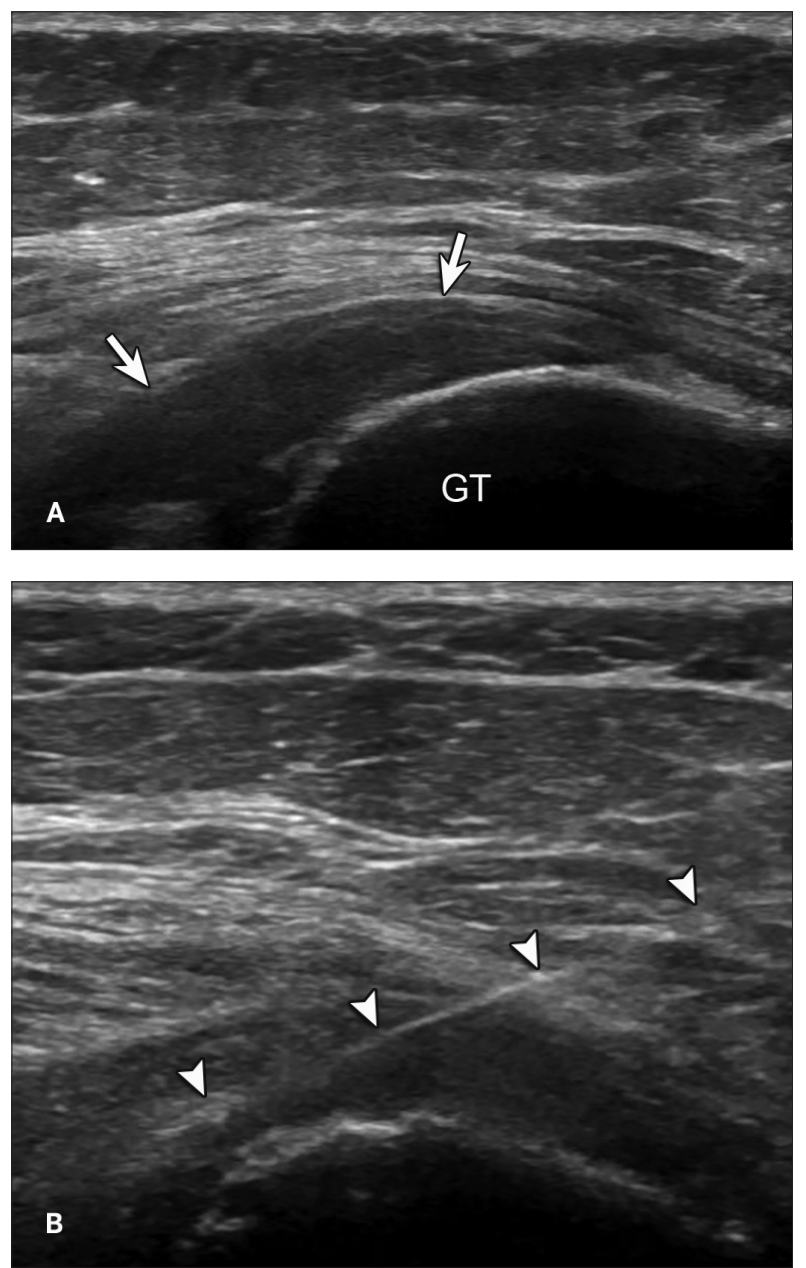
A more long-term assessment of patient symptoms was then completed. Retrospective chart review identified follow-up visits in 20 of the 21 patients, with an average interval of 42 months (range, 1-70 months). Of the 10 patients who initially showed marked improvement in symptoms after fenestration, clinic notes showed that 3 were asymptomatic. The remaining 7 patients received routine clinical care (health maintenance visits) with no description of hip symptoms or abnormal physical examination findings. Of the 8 patients with some improvement after fenestration, follow-up visits revealed that 2 were asymptomatic; 2 showed no change from earlier assessment with some improvement; and 4 had notes without any description of abnormal hip or pelvis signs or symptoms. Of the 2 patients who had no change in symptoms after the fenestration, 1 had an interval hip replacement and was pain free, and the other had clinic notes without mention of abnormal hip or pelvis signs or symptoms. Of the 2

Figure 2. Images from a 60-year-old woman with proximal hamstring tendinosis and fenestration. A, Sonogram in a long-axis orientation to the proximal hamstrings showing hamstring tendons $(\mathrm{H})$ with abnormal hypoechoic enlargement (arrows) at the ischial tuberosity (I) (right side of image is caudal). B, Sonogram in a short-axis orientation to the hamstrings showing a 20-gauge needle (arrowheads) with the distal tip in the tendon (left side of image is lateral).
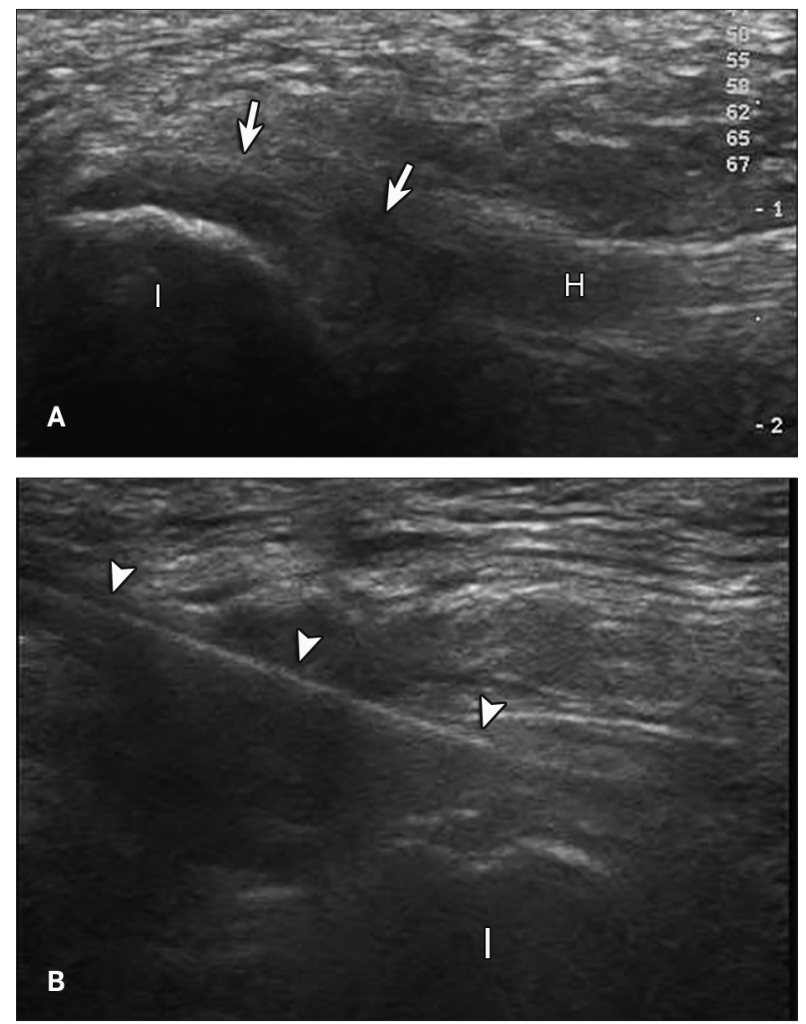

patients who were initially worse after the fenestration, both had been seen for routine health maintenance with no mention made of hip or pelvis signs or symptoms. In no case was a tendon tear or infection described or documented in the clinical notes. The completion of physical therapy or other exercise programs after fenestration could not be determined given the retrospective design of this study.

\section{Discussion}

Prior research has shown that ultrasound-guided tendon fenestration can be an effective treatment for tendinopathy. In particular, prior studies have focused primarily on the common extensor tendon of the elbow, the proximal patellar tendon, and the Achilles tendon. ${ }^{6}$ The results of this retrospective study show that ultrasound-guided tendon fenestration can be effective in the treatment of tendinopathy about the hip and pelvis, showing clinical improvement in $82 \%$.

The histologic findings of chronic tendinopathy have been shown to predominantly represent an underlying degenerative process (termed tendinosis), which includes mucoid degeneration, angiofibroblastic proliferation, and

Figure 3. Image from a 47-year-old man with gluteus minimus tendinosis and fenestration. Sonogram in a long-axis orientation to the gluteus minimus showing a 20-gauge needle (arrowheads) during fenestration with the distal tip in the abnormal hypoechoic and thickened gluteus minimus tendon at the anterior facet of the greater trochanter (GT) (left side of image is cephalad).

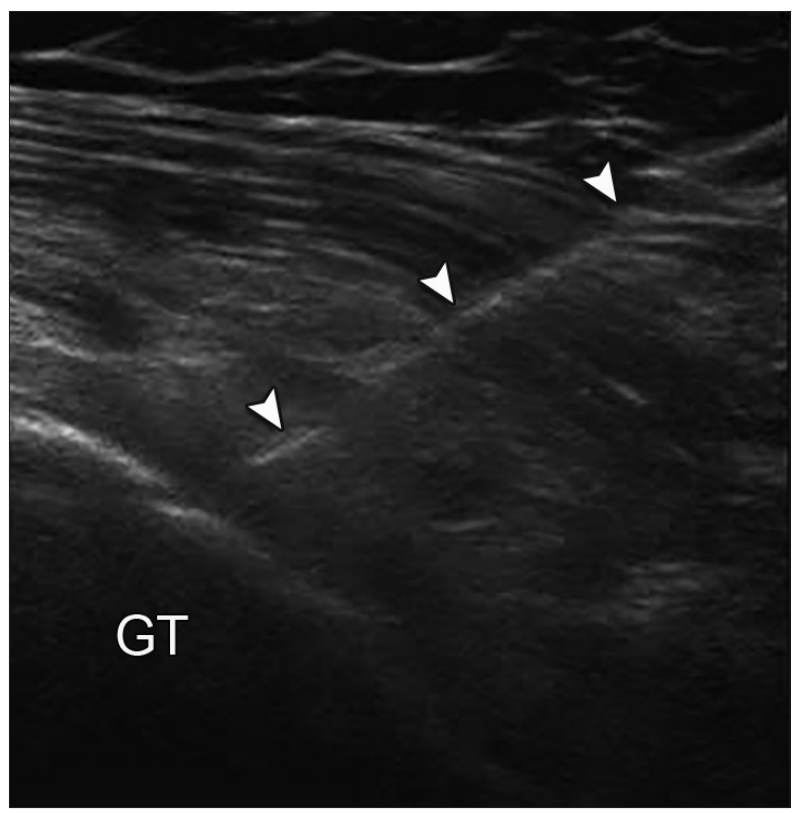


chondroid metaplasia. ${ }^{8-13}$ Interstitial and partial tendon tearing may also be a feature. Given the lack of a substantial inflammatory component, the term "tendinitis" is therefore not appropriate. Although inflammation does occur acutely after an injury, inflammation decreases by 10 days as the proliferative phase of healing begins and then becomes absent with chronic tendinopathy. ${ }^{14}$ Describing a condition by its correct term is important when considering appropriate tendon treatment.

Corticosteroid injection, either superficial to or within a tendon, has previously been considered as a treatment for chronic tendinopathy, largely because of a misunderstanding of the underlying tendon abnormality and the incorrect term "tendinitis" in this situation. ${ }^{5}$ The use of corticosteroid injection as an anti-inflammatory agent is questioned, as inflammation in chronic tendinopathy is not a prominent feature. Injection of a corticosteroid superficial

Figure 4. Images from a 62-year-old man with tensor fascia latae tendinosis and fenestration. A, Sonogram in a long-axis orientation to the tensor fascia latae showing abnormal hypoechoic enlargement of the proximal tensor fascia latae tendon (arrows) at the ilium (I). B, Twentygauge needle (arrowheads) during fenestration (left side of image is cephalad).
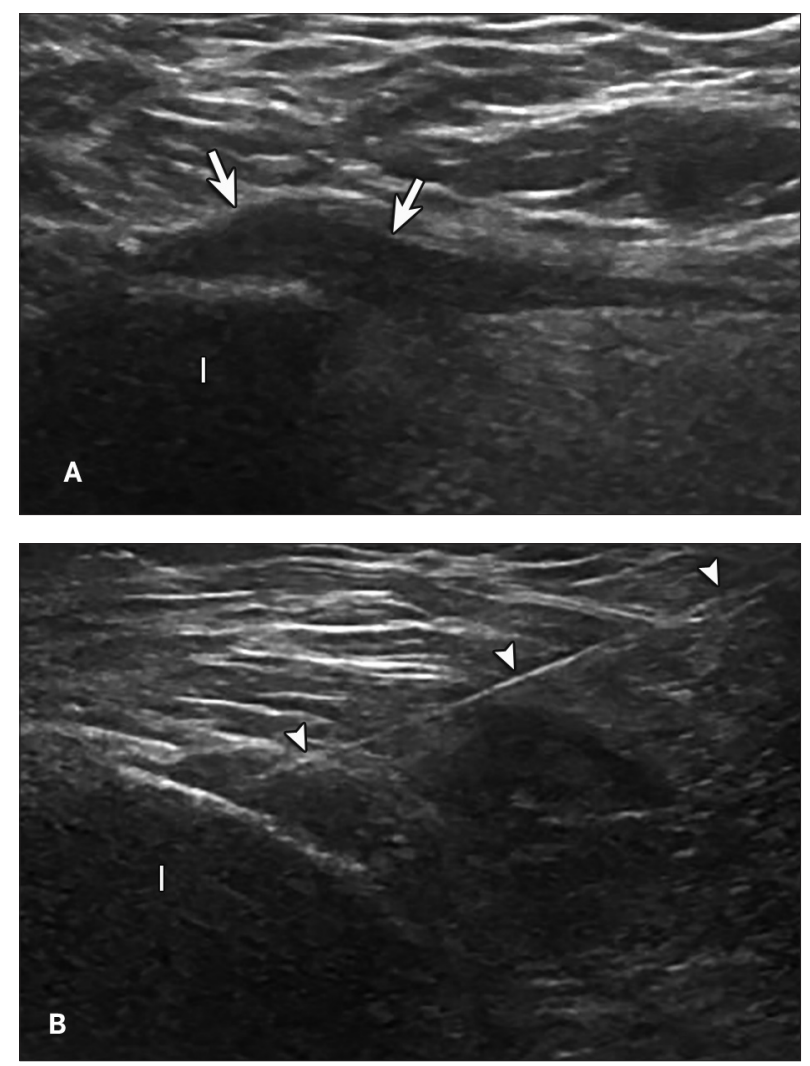

to tendinosis may have merit in some situations to provide temporary pain relief. ${ }^{5}$ Although the exact mechanism of pain relief is controversial, such temporary pain relief may allow the patient to complete eccentric strengthening as a noninvasive treatment for chronic tendinopathy. Of note, symptoms are expected to return after corticosteroid injection, and in the case of the common extensor tendon of the elbow, the pain will likely be more severe than before the injection. ${ }^{5,15}$ Given the questionable use and only temporary effects of corticosteroid injection, other percutaneous tendon treatments have been explored. In addition, McShane et $\mathrm{al}^{16}$ have shown that the addition of corticosteroid injection in conjunction with ultrasound-guided tenotomy did not improve patient outcomes. In our study, there was no significant difference in patient outcomes after tendon fenestration when patients had prior corticosteroid injection.

Tendon fenestration, also termed tenotomy or dry needling, has been used for nearly 2 decades. ${ }^{7}$ The use of ultrasound in conjunction with tendon fenestration has the benefits of accurately guiding the needle to the abnormal tendon segment for treatment. The goal of tendon fenestration is to convert a chronic tendon abnormality into an acute condition to allow improved healing. ${ }^{4}$ The abnormal area of the tendon is targeted with the needle, causing tendon trauma, bleeding, and a subsequent increase in local growth factors and other substances to promote healing. ${ }^{4}$ These local changes cause inflammation of the tendon, which is the initial phase of tissue healing, lasting up to 7 to 10 days after the procedure. ${ }^{14}$ This factor is the reason why anti-inflammatory medications and ice are avoided during this initial recovery period, so that the healing cascade is not potentially dampened. The next several weeks constitute the period of tissue proliferation, in which excessive stress on the tendon is avoided. ${ }^{14}$ Although these postprocedural guidelines have not been proven essential in the literature, it is believed that following such guidelines will allow the best chance for tissue healing.

One of the first descriptions of ultrasound-guided tenotomy was its use for treatment of Achilles tendinopathy as an alternative to surgery after failure of conservative management. ${ }^{17,18}$ Ultrasound-guided tenotomy of the common extensor tendon of the elbow has also been reported. ${ }^{7}$ These results showed that the procedure was safe and effective, with $85.5 \%$ of patients indicating that they would refer a friend or relative for a similar procedure. ${ }^{7}$ A study of patellar tendon fenestration demonstrated that in 47 patellar tendons in 32 patients, $81 \%$ had an excellent or good satisfaction score. ${ }^{19}$ Assessment of fenestration of other tendons about the body has not been extensively evaluated. One prior study described fenestration of the 
gluteus medius and hamstring in 2 patients; however, a more extensive evaluation of the hip and pelvis tendons has not been completed. ${ }^{6}$ The results of our study show that ultrasound-guided fenestration of 22 tendons about the hip and pelvis resulted in marked improvement in $45.5 \%$ and some improvement in $36.4 \%$. A more long-term follow-up assessment using clinic notes (average of 42 months after fenestration) showed that none of the patients had worsening symptoms described in clinical notes beyond the initial assessment. On the contrary, only 3 of the 21 patients had continued symptoms, with the others either being asymptomatic or not discussing their symptoms during routine follow-up clinic visits.

The gluteus medius was the most commonly treated tendon in this study, representing $50 \%$ of our patients, with most showing tendinosis. This finding is consistent with reports in the literature that the gluteus medius is commonly abnormal, with greater trochanteric pain syndrome. ${ }^{10,20}$ Of note, none of the patients in our study group had bursal distention in the region of the greater trochanter, a finding that is also consistent with prior studies indicating that bursal distention and true bursitis are uncommon findings in greater trochanteric pain syndrome. ${ }^{20,21}$ The next most common tendon treated was the proximal hamstring. In 2 of our patients, calcification was present in the area of tendinosis. When evaluating the specific tendon and fenestration outcomes, we found no trend to indicate that fenestration of one tendon was more likely to be successful than fenestration of another tendon. In our study, only $9.1 \%$ of patients ( 2 of 22 ) had a negative outcome (1 gluteus medius and 1 proximal hamstring). There were no clinical variables (patient age, sex, specific tendon, chronicity of symptoms, prior physical therapy, or prior corticosteroid injection) or ultrasound findings (tendinosis versus partial tear) that were significantly different between the group that did not have a positive outcome and the group that responded favorably. Although other ultrasound variables, such as the extent of the tendon abnormality and flow on color or power Doppler imaging, were not assessed in this study, a prior study by Kanaan et $\mathrm{al}^{22}$ has shown that the only ultrasound finding that was a positive predictive finding for successful ultrasound-guided tendon fenestration was a well-defined tendon abnormality; the presence or absence of hyperemia on Doppler imaging was not a predictive finding.

Further types of percutaneous tendon treatments have been described, such as injection of a hyperosmolar dextrose solution, autologous whole blood, and plateletrich plasma. ${ }^{4}$ Of note, tendon fenestration is typically performed with each of these other percutaneous tendon treatments. Although a comprehensive review of these tendon treatments is not relevant to this study, it is important to briefly review the studies in which tendon fenestration alone has been compared to other percutaneous treatments. A study by Dragoo et al ${ }^{23}$ comparing ultrasound-guided fenestration to platelet-rich plasma for treatment of patellar tendinopathy showed no significant difference in clinical outcomes at 26 weeks after treatment. In addition, studies comparing platelet-rich plasma to intratendon saline injection for treatment of tendinopathy of the common extensor tendon and Achilles tendon each showed no significant differences in clinical outcomes. ${ }^{24,25}$ Further studies are still required to determine whether fenestration alone is adequate for treatment of chronic tendinopathy and if one treatment versus another is more appropriate for tendinosis versus tendinosis with a superimposed tendon tear.

We acknowledge several imitations to this study. Given the retrospective nature, we had to rely on dictated follow-up visit notes from the referring clinicians to determine whether symptoms had improved. A prospective trial would allow more specific and objective assessment of patient symptoms at uniform intervals. In addition, imaging follow-up was not obtained, so it is unclear whether changes on ultrasound imaging occurred after the tendon fenestration and whether such changes correlated with patient outcomes. Last, we did not compare tendon fenestration to other treatments; a blinded randomized controlled trial would be important to provide such information.

In conclusion, the results of our study show that ultrasound-guided tendon fenestration can be effective in the treatment of tendinopathy about the hip and pelvis, showing clinical improvement in $82 \%$. Further studies are required to determine the long-term effects of ultrasoundguided tendon fenestration and to compare fenestration to other percutaneous tendon treatments.

\section{References}

1. Bancroft LW, Blankenbaker DG. Imaging of the tendons about the pelvis. AJR Am J Roentgenol 2010; 195:605-617.

2. Kingzett-Taylor A, Tirman PF, Feller J, et al. Tendinosis and tears of gluteus medius and minimus muscles as a cause of hip pain: MR imaging findings. AJR Am J Roentgenol 1999; 173:1123-1126.

3. Lustenberger DP, Ng VY, Best TM, Ellis TJ. Efficacy of treatment of trochanteric bursitis: a systematic review. Clin J Sport Med 2011;21:447453.

4. Chiavaras MM, Jacobson JA. Ultrasound-guided tendon fenestration. Semin Musculoskelet Radiol 2013; 17:85-90. 
5. Coombes BK, Bisset L, Vicenzino B. Efficacy and safety of corticosteroid injections and other injections for management of tendinopathy: a systematic review of randomised controlled trials. Lancet 2010; 376:17511767.

6. Housner JA,Jacobson JA, Misko R. Sonographically guided percutaneous needle tenotomy for the treatment of chronic tendinosis.J Ultrasound Med 2009; 28:1187-1192.

7. McShane JM, Nazarian LN, Harwood MI. Sonographically guided percutaneous needle tenotomy for treatment of common extensor tendinosis in the elbow. J Ultrasound Med 2006; 25:1281-1289.

8. Astrom M, Gentz CF, Nilsson P, Rausing A, Sjoberg S, Westlin N. Imaging in chronic Achilles tendinopathy: a comparison of ultrasonography, magnetic resonance imaging and surgical findings in 27 histologically verified cases. Skeletal Radiol 1996; 25:615-620.

9. Buck FM, Grehn H, Hilbe M, Pfirrmann CW, Manzanell S, Hodler J. Magnetic resonance histologic correlation in rotator cuff tendons.J Magn Reson Imaging 2010; 32:165-172.

10. Fearon AM, Scarvell JM, Cook JL, Smith PN. Does ultrasound correlate with surgical or histologic findings in greater trochanteric pain syndrome? A pilot study. Clin Orthop Relat Res 2010; 468:1838-1844.

11. Khan KM, Bonar F, Desmond PM, et al. Patellar tendinosis (jumper's knee): findings at histopathologic examination, US, and MR imaging. Victorian Institute of Sport Tendon Study Group. Radiology 1996; 200:821-827.

12. Kjellin I, Ho CP, Cervilla V, et al. Alterations in the supraspinatus tendon at MR imaging: correlation with histopathologic findings in cadavers. Radiology 1991; 181:837-841.

13. Potter HG, Hannafin JA, Morwessel RM, DiCarlo EF, O’Brien SJ, Altchek DW. Lateral epicondylitis: correlation of MR imaging, surgical, and histopathologic findings. Radiology 1995; 196:43-46.

14. Lee KS, Wilson JJ, Rabago DP, Baer GS, Jacobson JA, Borrero CG. Musculoskeletal applications of platelet-rich plasma: fad or future? AJR Am J Roentgenol 2011; 196:628-636.

15. Coombes BK, Bisset L, Brooks P, Khan A, Vicenzino B. Effect of corticosteroid injection, physiotherapy, or both on clinical outcomes in patients with unilateral lateral epicondylalgia: a randomized controlled trial. JAMA 2013; 309:461-469.

16. McShane JM, Shah VN, Nazarian LN. Sonographically guided percutaneous needle tenotomy for treatment of common extensor tendinosis in the elbow: is a corticosteroid necessary? J Ultrasound Med 2008; 27:11371144.

17. Testa V, Capasso G, Benazzo F, Maffulli N. Management of Achilles tendinopathy by ultrasound-guided percutaneous tenotomy. Med Sci Sports Exerc 2002; 34:573-580.

18. Testa V, Capasso G, Maffulli N, Bifulco G. Ultrasound-guided percutaneous longitudinal tenotomy for the management of patellar tendinopathy. Med Sci Sports Exerc 1999; 31:1509-1515.

19. Housner JA, Jacobson JA, Morag Y, Pujalte GG, Northway RM, Boon TA. Should ultrasound-guided needle fenestration be considered as a treatment option for recalcitrant patellar tendinopathy? A retrospective study of 47 cases. Clin J Sport Med 2010; 20:488-490.
20. Long SS, Surrey DE, Nazarian LN. Sonography of greater trochanteric pain syndrome and the rarity of primary bursitis. AJR Am J Roentgenol 2013; 201:1083-1086.

21. Silva F, Adams T, Feinstein J, Arroyo RA. Trochanteric bursitis: refuting the myth of inflammation. J Clin Rheumatol 2008; 14:82-86.

22. Kanaan Y,Jacobson JA,Jamadar D, HousnerJ, CaoiliEM. Sonographically guided patellar tendon fenestration: prognostic value of preprocedure sonographic findings. J Ultrasound Med 2013; 32:771-777.

23. Dragoo JL, Wasterlain AS, Braun HJ, Nead KT. Platelet-rich plasma as a treatment for patellar tendinopathy: a double-blind, randomized controlled trial. Am J Sports Med 2014; 42:610-618.

24. de Jonge S, de Vos RJ, Weir A, et al. One-year follow-up of platelet-rich plasma treatment in chronic Achilles tendinopathy: a double-blind randomized placebo-controlled trial. Am J Sports Med 2011; 39:16231629

25. Krogh TP, Fredberg U, Stengaard-Pedersen K, Christensen R, Jensen P, Ellingsen T. Treatment of lateral epicondylitis with platelet-rich plasma, glucocorticoid, or saline: a randomized, double-blind, placebo-controlled trial. Am J Sports Med 2013; 41:625-635. 